Stawomir Śledziewski

Diocesan Seminary in Łomża

Cardinal Stefan Wyszyński University in Warsaw, Poland

\title{
Die Bedeutung Johannes Pauls II. in der Auseinandersetzung zwischen Theologie und Naturwissenschaften
}

\section{The Importance of John Paul II in the Conflict between Theology and Science}

\begin{abstract}
One of the characteristics of the teaching of Pope John Paul II is his approach to the subject of faith and reason, which have played a central role in the development of civilization. They work best when they mutually challenge one another to look farther, to probe more deeply, in quest of truth. Hostility between faith and reason is not accepted because both have their source in God. But when both work together, the human person has the chance of discovering the full truth about himself, the world and God. The author of this article argues that a large number of papal documents and pronouncements of Pope John Paul II show his contribution towards the bringing together of natural sciences and theology. This is also a challenge for those who continue this effort.
\end{abstract}

Keywords

John Paul II, faith and reason, science and theology, "Fides et ratio".

\section{Einleitung}

Papst Johannes Paul II. gilt als derjenige, der die Kirche Christi in das III. Jahrtausend eingeführt hat. Dies ist nicht nur eine geschichtliche Tatsache, sondern viel mehr hat es eine umfassende Bedeutung für heutige Zeit. Ängste und Ungewissheit, die die gegenwärtige Menschheit begleiten, wurden durch 
seine Hoffnung und sein großes Vertrauen in die Vorsehung Gottes gemindert. Seine Amtszeit von über 26 Jahren prägte nachhaltig viele Menschen in der ganzen Welt. Darum hat ihn die amerikanische Zeitschrift „Time“ zu einer der zwanzig einflussreichsten Personen des XX. Jahrhunderts gewählt. Zugleich hat ihn diese Zeitschrift zum Mann des Jahres 1994 gekürt, als zweiten Papst, neben Johannes XXIII. im Jahre 1962. Im Jahr 2003 war Johannes Paul II. für den Friedensnobelpreis nominiert.

\section{Enzyklika „Fides et ratio“ als Wegweiser der Relation zwischen Vernunft und Glaube}

Was hat zu dem Phänomen Johannes Paul II. beigetragen? Warum haben Menschen aus der ganzen Welt ihn als Vorbild und Autorität nicht nur im religiösen Bereich gesehen? Eine mögliche Antwort auf diese Fragen wäre mit Sicherheit, dass Karol Wojtyła jedem Menschen mit Würde begegnete. Dazu kommt die klare Einstellung gegenüber der einheitlichen Philosophie der menschlichen Rechte und Werte. Mit dieser „Menschlichkeit“ haben wir in vielen Werken von Johannes Paul II. zu tun. Die wichtigsten Publikationen des Heiligen Vaters sind seine Enzykliken. Die dreizehnte Enzyklika trägt den Titel „Fides et ratio“ und wurde am 14.09.1998 veröffentlicht. Sie beschäftigt sich mit der Relation zwischen Glauben und Vernunft und beinhaltet zugleich eine Einladung an alle Menschen, die sich mit der Problematik auseinandersetzen, sowohl ihren Glauben als auch ihre Vernunft zu vertiefen. Diese Enzyklika setzte ein wichtiges Zeichen für den interdisziplinären Dialog zwischen Naturwissenschaften und Theologie mit der Philosophie. Dieser Dialog brauchte neue Impulse und eine Korrektur, weil sich in den letzten vierhundert Jahren viele Unstimmigkeiten, Missverständnisse und sogar Aggressionen angesammelt haben. Dieser Dialog soll sich vor allem in Bereichen abspielen, wo er bis heute an gegenseitiger Ignoranz, sprachlicher Unschärfe sowie an fundamentalistischen Tendenzen scheitert. Die gegenseitige Ergänzung der Theologie und der Naturwissenschaften soll zu einer endgültigen Überwindung jener Bewusstseins- und Gewissensspaltung beitragen, die seit der Zeit des Galileo Galilei (1564-1642) die geistige Dimension unserer Welt immer noch belasten.

In diesem Zusammenhang hat Johannes Paul II. immer wieder die veränderte und positive Einstellung der Kirche gegenüber den Naturwissenschaften betont: „Zwischen einer Vernunft, welche durch ihre gottgegebene Natur auf Wahrheit angelegt und zur Erkenntnis der Wahrheit befähigt ist, und dem Glauben, der 
sich der gleichen göttlichen Quelle aller Wahrheit verdankt, kann es keinen grundsätzlichen Konflikt geben. Der Glaube bestätigt gerade das Eigenrecht der natürlichen Vernunft. Er setzt es voraus" ${ }^{\text {"1 }}$. Papst Johannes Paul II. wollte in erster Linie, dass die Kirche das oft mit Vorurteilen behaftete Denken aufgibt. Gleichzeitig schien ihm bewusst zu sein, dass die Glaubwürdigkeit der Kirche und ihre Rolle für den modernen Menschen ihre Bedeutung aufs Neue gewinnen kann, wenn die Errungenschaften der Naturwissenschaften akzeptiert werden. Andererseits sollen sich die Naturwissenschaften darüber im Klaren sein, dass sie selbstkritisch sein müssen und innerhalb ihrer Deutungsansprüche die Sinnfrage nicht beantworten können. Diese Einstelllung hilft ihnen, sich gegenüber der Theologie viel freier zu verhalten. Die Kenntnis der Problematik der letzten Jahrhunderte im Bereich der Relation zwischen Theologie und Naturwissenschaften bewegte den polnischen Papst eine „richtige“ Stellung der Vernunft im Glauben und des Glaubens in der Vernunft aufzuzeigen. Johannes Paul II. hatte nicht nur keine Angst vor der Gegenüberstellung der beiden Bereiche, sondern er wusste im Gegenteil, dass erst in ihrer Harmonie der Mensch im Stande wäre, einen Zugang zu der Wahrheit über sich selbst, über die Welt und über Gott zu finden.

„Alle bitte ich, sich intensiv um den Menschen, den Christus im Geheimnis seiner Liebe gerettet hat, und um sein ständiges Suchen nach Wahrheit und Sinn zu kümmern. Verschiedene philosophische Systeme haben ihn durch Täuschung überzeugt, dass er sein absolut eigener Herr sei, der autonom über sein Schicksal und seine Zukunft entscheiden könne, wenn er ausschließlich auf sich selbst und seine Kräfte vertraut. Das wird niemals die Größe des Menschen ausmachen können. Bestimmend für seine Verwirklichung wird nur die Entscheidung sein, sich dadurch in die Wahrheit einzufügen, daß er im Schatten der Weisheit seine Wohnung errichtet und in ihr wohnen bleibt. Erst in diesem Wahrheitshorizont wird er begreifen, wie sich seine Freiheit im Vollsinn entfaltet und daß er zur Liebe und zur Erkenntnis Gottes berufen ist. Darin liegt seine höchste Selbstverwirklichung“"2.

Dieses Zitat aus der Enzyklika „Fides et ratio“ - des wichtigsten Dokuments Johannes Pauls II. über die Relation von Vernunft und Glaube - zeigt einen tiefen Sinn der Wahrheit, nach der jeder Mensch, der sich die Grundfragen im Bereich der Existenz stellt und sich Gedanken über den Sinn seinen Lebens macht, streben soll ${ }^{3}$.

\footnotetext{
${ }^{1}$ Papst Johannes Paul II., Ansprache an Wissenschaftler und Studenten im Kölner Dom am 15. November 1980.

${ }^{2}$ Johannes Paul II., Fides et ratio, Rom 1998, Nr. 107.

${ }^{3}$ Vgl. Johannes Paul. II., Przekroczyć próg nadziei (Die Schwelle der Hoffnung überschreiten), Lublin 1994, S. 41nn.
} 
Diese Wahrheit wird im Kontext der Schöpfungslehre als Offenbarungswahrheit und in den Naturwissenschaften als Naturwahrheit bezeichnet. Diese beiden Wahrheiten können nicht in einem Widerspruch zueinander stehen, weil derselbe Gott, „der die Verstehbarkeit und Vernünftigkeit der natürlichen Ordnung der Dinge, auf die sich die Wissenschaftler vertrauensvoll stützen, begründet und gewährleistet, ist identisch ist mit dem Gott, der sich als Vater unseres Herrn Jesus Christus offenbart" ${ }^{\text {"4 }}$ Um das zu verstehen sollen wir nach Johannes Paul II. den Prozess des Dialogs zwischen Theologie und Naturwissenschaften akzeptieren, unterstützen und entfalten. Erst dann kann der Mensch der Wahrheit näher kommen, wenn er mehr und mehr die Wirklichkeit zu verstehen versucht, als auch sich selbst und all das, was seine Existenz betrifft. In diesem Zusammenhang wies Johannes Paul II. auf die personalistische Dimension der menschlichen Vernunft hin: „Einerseits erscheint die Erkenntnis durch Glauben als eine unvollkommene Erkenntnisform, die sich nach und nach durch die persönlich gewonnene Einsicht vervollkommnen soll; andererseits erweist sich der Glaube oft als menschlich reicher im Vergleich zur bloßen Einsichtigkeit, weil er eine Beziehung zwischen Personen einschließt und nicht nur die persönlichen Erkenntnisfähigkeiten, sondern auch die tiefergehende Fähigkeit ins Spiel bringt, sich anderen Personen anzuvertrauen, indem man eine festere und innige Verbindung mit ihnen eingeht" “5. Das Verhältnis zwischen „fides“ und „ratio“ soll immer dem Menschen dienen und darum stand der Mensch im Zentrum der Überlegungen und der Sorge von Johannes Paul II. Im Konflikt dieser beiden Bereiche sah Wojtyła eine der größten und der destruktivsten Tragödien des Menschen.

\section{Geschichtlicher Ausblick und Aussagen über die Problematik des Verhältnisses zwischen Theologie und Naturwissenschaften Karol Wojtyłas und Papsts Johannes Pauls II. bis zur Enzyklika „Fides et ratio“}

Karol Wojtyła hat sich bereits als junger Priester sehr für die Problematik der Relation zwischen Theologie und Naturwissenschaften interessiert. Dieses Interesse begann im Jahre 1953, als Wojtyła mit zwei Atomphysikern zum Skifahren in das Tatragebirge reiste. Der Ausflug eröffnete eine Reihe von philosophisch-naturwissenschaftlichen Diskussionen, die mit der Zeit zu einem

\footnotetext{
${ }^{4}$ Johannes Paul II., Fides et ratio 34.

${ }^{5}$ Johannes Paul II., Fides et ratio 32.
} 
Zyklus von Seminaren im Bereich Glauben-Naturwissenschaften anwuchsen ${ }^{6}$. Karol Wojtyła gebrauchte als Philosoph und Humanist Argumente aus dem Bereich der Humanwissenschaften und Philosophie. Seine Gesprächspartner versuchten ihre Standpunkte zu zeigen, die aus der Methode der Naturwissenschaften stammten. Für den späteren Papst hatten diese Gespräche und Begegnungen einen komplementären Charakter, den er immer wieder betonte. Als Bischof und Kardinal von Krakau nahm er gerne an diesen interdisziplinären Disputen teil. Sie prägten seine Meinung über den Dialog und die interdisziplinäre Problematik.

Als das Zweite Vatikanische Konzil im Jahre 1962 begann, nahm Karol Wojtyła aktiv an den Sitzungen teil. Für den jungen Weihbischof von Krakau war das Vaticanum II. ein „großes Miterleben der Kirche“ oder wie man zu sagen pflegte ein "Seminar des Heiligen Geistes“7 . Nach vielen Jahren bereits als Papst hat Wojtyła im Gespräch mit André Frossard über das Zweite Vatikanische Konzil gesagt, dass es „ein Ereignis war, welches mir aus der Perspektive meines eigenen Glaubens verhalf, eine volle Synthese zu finden“" . Der Weihbischof aus Krakau hat stark zur endgültigen From der Konstitution Lumen Gentium beigetragen, die neben der Konstitution Gaudium et spes zum bedeutungsvollen Dokument des Konzils wurde. Die Konstitution Gaudium et spes verdankt ihre endgültige Fassung auch Wojtyła, der nach der Vorstellung der Änderungen seitens der polnischen Bischofskonferenz zum Mitglied der Zentralen Unterkommission für dieses Dokument wurde9 Der Text der Konstitution will eine neue tiefe und vielseitige Vorstellung der Anwesenheit der Kirche und der Christen in der gegenwärtigen Zeit und Welt darbieten. Sie spricht von „Zwei verschiedenen Erkenntnisordnungen“, Glaube und Vernunft, und bezieht sich auf die Dogmatische Konstitution Dei Filius über den katholischen Glauben des Ersten Vatikanischen Konzils ${ }^{10}$. Die Botschaft der Pastoralkonstitution ist in unserem Zusammenhang eindeutig, wenn ,die methodische Forschung in allen Wissensbereichen in einer wirklich wissenschaftlichen Weise und gemäß den Normen der Sittlichkeit vorgeht, wird sie niemals in einen echten Konflikt mit dem Glauben kommen, weil die Wirklichkeiten des profanen Bereichs und die des Glaubens in demselben Gott

${ }^{6}$ Vgl. Johannes Paul II., Nauka wobec problemów człowieka, in: Johannes Paul II., Wiara i kultura (Glaube und Kultur), Rom 1986, S. 35.

${ }^{7}$ Johannes Paul II., Die Schwelle der Hoffnung überschreiten, Lublin 1994, S. 125.

${ }^{8}$ A. Frossard, Nie lękajcie się! Rozmowy z Janem Pawłem II, Libreria Editrice Vaticana 1982, S. 86.

${ }^{9}$ Vgl. T. Szulc, Jan Pawet II, Warszawa 1996, S. 219.

${ }^{10}$ Gaudium et spes, in: Sobór Watykański II. Konstytucje. Dekrety. Deklaracje, Poznań 1967, S. 587. 
ihren Ursprung haben"11. Glaube und Vernunft sind Elemente der Relation zwischen der entdeckenden Wahrheit durch die Naturwissenschaften und der erkannten Wahrheit dank der übernatürlichen Offenbarung. Zwischen ihnen kann es nicht zu einem Gegensatz kommen. Dieser Meinung war auch Karol Wojtyła, der immer mehr davon ausging, dass die Auseinandersetzung zwischen den beiden Realitäten, die seit dem sog. „Fall Galilei“ vorhanden war, nun zum Dialog zur Wiederannäherung kommen sollte. Darum sprach er sich zusammen mit Kardinal König aus Wien während des Zweiten Vatikanischen Konzils für eine Neuregelung der Relation des Problems zwischen Theologie und Naturwissenschaften aus ${ }^{12}$. Den Konzilsvätern schienen dennoch andere Themen viel relevanter für die damalige Kirche und sie bezogen vorerst nicht zu dieser Auseinandersetzung Stellung.

Erst mit dem Pontifikat Karol Wojtyłas als Johannes Paul II. wurde diese Problematik wieder aufgegriffen. Dies beweisen viele Stellen in seinen Ansprachen (z. B.: Ansprache an die Mitglieder der "European Physical Society" (30. März 1979); Zum Gedenken an Albert Einstein anlässlich des Jahrestags seiner Geburt (10. November 1979); An eine Gruppe von Nobelpreisträgern (22. Dezember 1980) ${ }^{13}$. Johannes Paul II griff dieses Thema auch in der Ansprache an Wissenschaftler und Studenten im Kölner Dom am 700. Todestag des hl. Albertus Magnus, am 15. November 1980 auf. Im Bezug auf die Person des hl. Albertus Magnus, der als Bischof und zugleich Gelehrter die notwendige Unterscheidung und Beziehung der beiden Erkenntnisordnungen von Glaube und Vernunft vertreten hatte betonte der Papst, dass eine Wissenschaft, die sich auf Vernunftgründe stützt und methodisch gesichert fortschreitet, nicht zu Erkenntnissen gelangt, die in Konflikt mit der Glaubenswahrheit kommen ${ }^{14}$. Deswegen darf man die Wissenschaft nicht gleichgültig behandeln. Andererseits ist der Glaube für die Wissenschaft eine Notwendigkeit. Gerade er beschützt vor den Gefahren, die aus den Manipulationen innerhalb der Wissenschaft entstehen und sich hinter dem wissenschaftlichen Fortschritt verstecken. Vor allem ist hier die Dehumanisierung gemeint. Als erstes Kriterium, das über den wissenschaftlichen

\footnotetext{
${ }^{11}$ Johannes Paul II., Fides et ratio 36.
}

${ }^{12}$ Vgl. T. Szulc, Jan Pawet II, Warszawa 1996, S. $211 \mathrm{n}$.

${ }^{13}$ Vgl. All diese Ansprachen aus den ersten Jahren des Pontifikats befinden sich im Buch Johannes Paul II, Wiara i kultura (Glaube und Kultur), Rom 1986.

${ }^{14}$ Vgl. Johannes Paul II., Ansprache an Wissenschaftler und Studenten im Kölner Dom am 700. Todestag des Hl. Albertus Magnus, am 15. November 1980, Nr. 3. 
Fortschritt bestimmt, soll der Dienst am dem ganzen Menschen sein, in seiner ganzheitlichen sowohl geistigen als auch materiellen Realität ${ }^{15}$.

All diese und andere päpstlichen Dokumente beweisen, dass sich mit dem Pontifikat Johannes Pauls II. ein Durchbruch in dem Dialog zwischen Wissenschaften und Theologie ereignet hat. Zugleich bleibt er eine Aufgabe für die Nachfolger.

Johannes Paul II. war kein Spezialist im Bereich der Naturwissenschaften oder der Naturphilosophie, aber er schätzte und bezog sich oft auf die Arbeit der führenden Gelehrten aus diesen Bereichen. Dem Streben des Menschen nach der Erkenntnis der Wahrheit über Gott, über den Menschen und über die Welt war all sein Tun untergeordnet, damit man diese Erfahrungen am besten gebrauchen kann. Wenn die Wahrheit zum Dreh- und Angelpunkt im Verhältnis von Theologie und Naturwissenschaften wird, dann können beide Bereiche ohne irgendwelche Hindernisse zusammenwirken, um den Menschen in seiner gesamten Situation in der Gegenwart zu bereichern.

In der Vielfalt der Dokumente, die Johannes Paul II. im Rahmen der Auseinandersetzung zwischen Theologie und Naturwissenschaften herausgegeben hat, bekommen vier eine Sonderstellung. Das erste davon wurde bereits oben erwähnt. Es handelt sich um die Ansprache zum Gedenken an Albert Einstein anlässlich des Jahrestags seiner Geburt (10. November 1979). Bei dieser Ansprache äusserte der Heilige Vater den Wunsch, eine Kommission zum Fall Galilei einzuberufen: „Ähnliche Anliegen hatte ich am 10. November 1979 aus Anlass der ersten Jahrhundertfeier seit der Geburt von Albert Einstein, als ich vor dieser gleichen Akademie den Wunsch aussprach, dass Theologen, Gelehrte und Historiker, vom Geist ehrlicher Zusammenarbeit beseelt, die Überprüfung des Falles Galilei vertiefen und in aufrichtiger Anerkennung des Unrechts, von welcher Seite es auch immer gekommen sein mag, das Misstrauen beseitigen, das dieses Ereignis noch immer bei vielen gegen eine fruchtbare Zusammenarbeit von Glaube und Wissenschaft, von Kirche und Welt hervorruft“. Zwei Jahre später wurde die Untersuchungskommission einberufen. Das zweite wichtige Dokument war das Schreiben an Pater George V. Coyne, Direktor der Vatikanischen Sternwarte „Specola“ (1. Juni 1988). Das dritte war die Botschaft an die Teilnehmer der Vollversammlung der Päpstlichen Akademie der Wissenschaften (22. Oktober 1996) über die Evolution. Das letzte und zugleich wichtigste Dokument war die Enzyklika „Fides et ratio“ aus dem Jahre 1998. Mit dieser Enzyklika, an welcher der Papst 13 Jahre gearbeitet hatte, wollte er die Bedeutung des menschlichen

${ }^{15}$ Vgl. Johannes Paul II., Ansprache an Wissenschaftler und Studenten im Kölner Dom am 700. Todestag des Hl. Albertus Magnus, am 15. November 1980, Nr. 3. 


\section{The Person and the Challenges \\ 100 Volume 2 (2012) Number 1}

Verstandes zeigen und seine Werte im Kontext der schwierigen geschichtlichen Bedingungen, die im Liberalismus und Postmodernismus diese Werte verworfen haben, verteidigen. Der Verstand trägt dazu bei, dass der Glaube reifer und tiefer wird. Dagegen gibt der Glaube dem menschlichen Leben einen Sinn und zeigt seine Ursache und sein Ziel ${ }^{16}$. Nach Johannes Paul II. kann es zwischen Glaube und Vernunft zu keinem Antagonismus kommen, weil beide ihren Ursprung in Gott haben. Diese Aussagen zeigen, dass der polnische Papst ein Mensch des Dialogs war. In dem Interview mit Vittorio Messori spricht er in diesem Zusammenhang folgende Worte: „Solchen Dialog (Dialog der Erlösung) sollen Christen nicht nur unter sich führen, sondern auch mit anderen nicht christlichen Religionen, mit der ganzen Welt der Kultur und Zivilisation, sogar auch mit denen, die nicht glauben. Die Wahrheit nämlich akzeptiert keine Grenzen. Sie ist für alle und für jeden. Und wenn man solche Wahrheit aus Liebe tut (vgl. Eph 4,15), dann wird sie noch mehr universalistisch" ${ }^{\text {"17. }}$. Diese Worte kann man als ein breit verstandenes päpstliches Programm auffassen. In diesem Programm versteht sich die Suche nach der Wahrheit als ein überragendes Paradigma. Darum befasst sich letztendlich die Enzyklika „Fides et ratio“ mit der Kernfrage vom Menschen überhaupt: die Frage nach der Wahrheit - ein unausweichliches und fundamentales Thema. Die italienische Presse würdigte diese Enzyklika als „Magna Charta des Denkens“.

Neben den vielen Dokumenten, die auf den Dialog zwischen Theologie und Naturwissenschaften hingewiesen haben, hat Johannes Paul II. auch ein anderes Zeichen der Offenheit gesetzt. Er rehabilitierte zuerst Galileo Galilei (1992), dann Nikolaus Kopernikus (1993) und schließlich Charles Darwin (1996). Der Tag der Rehabilitierung Darwins, der 22. Oktober 1996, wurde symbolisch zum Tag der geschichtlichen Beendigung des Streites zwischen der Kirche und den Naturwissenschaften. Johannes Paul II. wurde damit zum Sprachrohr des gegenseitigen Dialogs, der die Hindernisse der Isolation und Feindschaft zwischen Glaube und Vernunft überwindet. Der Papst rief zu einer radikalen Negation der Isolation, zur Befreiung vom gegenseitigen Misstrauen und zur Haltung der intellektuellen Öffnung auf, in der von Bedeutung ist, dass ,,jede Wissenschaft die andere bereichert und sie zur intellektuellen Haltung provoziert, damit sie in einer Fülle zu dem werden, was sie werden können und zu einer menschlichen Vision von dem, was wir sind und was wir werden, beitragen" ${ }^{\prime 18}$.

\footnotetext{
${ }^{16}$ Vgl. Johannes Paul II., Fides et ratio 42.

${ }^{17}$ Johannes Paul II., Die Schwelle der Hoffnung überschreiten, Lublin 1994, S. 126.

${ }^{18}$ Zagadnienia filozoficzne w nauce, XII, in: List Ojca Świętego Jana Pawła II do Ojca Georgea Coyne, S.J., Dyrektora Obserwatorium Watykańskiego, Kraków 1990, S. 2-12.
} 


\section{Ausblicke}

Mit dem Pontifikat Johannes Paul II wird die Auseinandersetzung zwischen Theologie und Naturwissenschaften zumindest seitens der Kirche eher zu einem Dialog. Dieser Dialog bekommt durch die Person Wojtyłas eine neue Qualität. Das Erbe von Johannes Paul II. im Bereich des Dialogs umfasst über 30 verschiedene Dokumente, die für uns eine Aufgabe darstellen, eine richtige Relation zwischen Theologie und Naturwissenschaften zu pflegen und zu entfalten. Dabei wird die Suche nach Kriterien des Dialogs wichtig. Darüber hinaus sieht der postmoderne Mensch immer deutlicher, dass es eine Notwendigkeit wird, den Dialog zu führen, in dem die Suche nach der Wahrheit, wie Johannes Paul II immer wieder betonte, und gegenseitiges Anerkennen nie vergessen werden sollen. Die Vielfalt der Aspekte des Dialogs und die durchaus positive Öffnung seitens der Kirche bilden in den Jahren nach dem Pontifikat Johannes Pauls II. eine weitere Möglichkeit der Verwirklichung des Dialogs, in dem die Klärung der Begriffe und die Reflexion über die Kriterien das künftige Miteinandersein von Theologie und Naturwissenschaften bestimmen werden ${ }^{19}$.

Diesen Artikel möchte ich abschließen mit einen Zitat aus der Enzyklika „Fides et ratio“, der eine Botschaft des Heiligen Vaters für die Zukunft enthält und zugleich ein Aufruf an die Jugend ist, dass sie ihr Leben auf dem richtigen Fundament aufbaue: „Denn man kann nicht leugnen, daß unsere Zeit mit ihren raschen und umfassenden Veränderungen vor allem die jungen Generationen, denen die Zukunft gehört und von denen sie abhängt, dem Gefühl aussetzt, ohne echte Bezugspunkte zu sein. Das Erfordernis eines Fundamentes, auf dem das Dasein des einzelnen und der Gesellschaft aufgebaut werden kann, macht sich vor allem dann in dringender Weise bemerkbar, wenn man die Bruchstückhaftigkeit von Angeboten feststellen muss, die unter Vortäuschung der Möglichkeit, zum wahren Sinn des Daseins zu gelangen, das Vergängliche zum Wert erheben. So kommt es, daß viele ihr Leben fast bis an den Rand des Abgrunds dahinschleppen, ohne zu wissen, worauf sie eigentlich zugehen. Das hängt auch damit zusammen, daß diejenigen, die dazu berufen waren, die Frucht ihres Nachdenkens in kulturellen Formen auszudrücken, den Blick von der Wahrheit abgewandt haben und der Mühe geduldigen Suchens nach dem, was gelebt zu werden verdient, den Erfolg im Unmittelbaren vorziehen“20.

${ }^{19}$ Vgl. A. Anderwald, Die Suche nach Kriterien. Zum Dialog zwischen Theologie und Naturwissenschaften gemäß der Lehre der Kirche, „Theologisches“ Jahrgang 39 (2009) Nr. 9/10, S. 318.

\footnotetext{
${ }^{20}$ Johannes Paul II., Fides et ratio 6.
} 


\section{Bibliography}

Anderwald A., Die Suche nach Kriterien. Zum Dialog zwischen Theologie und Naturwissenschaften gemäß der Lehre der Kirche, „Theologisches“, Jahrgang 39 (2009), Nr. 9/10, S. 306-318.

Johannes Paul II., Fides et ratio, Rom 1998.

Johannes Paul II., Przekroczyć próg nadziei (Die Schwelle der Hoffnung überschreiten), Lublin 1994.

Johannes Paul II., Wiara i kultura (Glaube und Kultur), Rom 1986.

Frossard A., Nie lękajcie się! Rozmowy z Janem Pawłem II, Libreria Editrice Vaticana 1982.

Szulc T., Jan Pawet II, Warszawa 1996.

Sobór Watykański II. Konstytucje. Dekrety. Deklaracje, Poznań 1967. 